\title{
Polymères à empreintes moléculaires pour l'extraction sélective de composés de milieux biologiques
}

\author{
Molecularly imprinted polymers for \\ selective extraction of analytes \\ from biological samples
}

Florence HUGON-CHAPUIS, Valérie PICHON

Laboratoire Environnement et Chimie Analytique (LECA), UMR CNRS 7121, École supérieure de Physique et de Chimie Industrielles (ESPCI) 10, rue Vauquelin - 75231 Paris Cedex 05

Tél : 0140794772 - Fax : 0140794776 - E-mail : florence.hugon@espci.fr

(Reçu le 25 juillet 2007 ; accepté après modification le 10 novernbre 2007)

\section{$R E ́ S U M E ́$}

L'analyse de composés d'intérêts présents dans les échantillons biologiques nécessite des méthodes de séparation et de détection performantes ainsi qu'une étape préalable de traitement de l'échantillon afin de préconcentrer l'analyte et de purifier la matrice.

La méthode de choix reste l'extraction sur phase solide (SPE), cependant, l'utilisation des supports conventionnels d'extraction mettent en jeu une rétention basée sur des interactions hydrophobes ce qui conduit fréquemment à la coextraction de composés de polarité similaire. Un apport en sélectivité au niveau de l'extraction peut être obtenu par des supports d'immunoaffinité basés sur l'utilisation d'anticorps spécifiques de l'analyte recherché gréffés sur un support solide (immunoadsorbants). Cependant, le coût et le temps nécessaires à la production d'anticorps ont récemment conduit à l'émergence d'une technique alternative basée sur la synthèse de polymères hautement réticulés possédant des cavités spécifiques d'une molécule modèle. Ces polymères à empreintes moléculaires (MIPs) possèdent des propriétés comparables à celles des immunoadsorbants en termes de sélectivité avec, en plus, l'avantage d'un cô̂t de développement réduit et d'une plus grande stabilité thermique et chi-

\section{SUMMARY}

The analysis of interest compounds from biological samples requires a procedure of pretreatment in order to preconcentrate the analytes and clean-up the matrix. Despite its attractive features, classical sorbents used in SPE retain analytes by non selective hydrophobic interactions that lead to a partial coextraction of interfering substances. So, sorbents displaying more selective interactions are powerful tools for the development of sensitive and more reliable methods.

Immunoaffinity supports can be used to obtain a selective extraction. They are based on the immobilization of specific antibodies developed against the target analyte (Inmunosorbent). Nevertheless, the cost and the time required to produce antibodies lead to the development of a new polymeric material: the Molecularly Imprinted Polymers (MIP). These supports possess molecular recognition sites designed for the analyte of interest. With performances similar to immunosorbents in terms of selectivity, MIPs present a better thermic and chemical stability and the cost of synthesis is also reduced. Like immunosorbents, their retention mechanism is based on molecular recognition. In most cases, the developed interactions during the extraction 
mique. Le mécanisme de rétention mis en jeu lors de l'étape d'extraction repose, comme pour les immnuoadsorbants, sur un mécanisme de reconnaissance moléculaire. Les interaclions mises en jeu lors de la reconnaissance sont pour la plupart des interactions non covalentes de type liaisons hydrogène, $\pi-\pi$ ou électrostatiques. Les polymères sont synthétisés en général sous forme de monolithe et utilisés soit en cartouche d'extraction ou directement intégrés dans le système d'analyse. Les nombreuses et récentes applications des MIPs pour des extractions à partir d'échantillons biologiques tels que le plasma ou l'urine ont permis de confirmer le réel potentiel de ces nouveaux supports synthétiques pour. l'extraction sélective de composés cibles.

\section{MOTS-CLÉS}

Polymères à empreintes moléculaires, Extraction sur phase solide, Sélectivité, Echantillons biologiques.

\section{Introduction}

Lors de l'analyse d'échantillons complexes, l'étape de traitement de l'échantillon est cruciale mais elle demeure pourtant longue à effectuer et peut être une possible source d'imprécisions pour la quantification finale du composé (1). Pendant de nombreuses années, l'extraction liquide-liquide a été la méthode de choix, elle est maintenant largement supplantée par l'extraction sur phase solide (SPE) qui permet la réduction de l'emploi de solvants et est mieux adaptée à l'extraction de composés polaires (2). Elle est fondée sur la distribution des composés entre une phase solide (adsorbant) et une phase liquide (échantillon). Cette méthode d'extraction repose en réalité sur un simple processus chromatographique dans lequel l'adsorbant joue le rôle de phase stationnaire et l'échantillon percolé constitue la phase mobile. Les solutés présents dans l'échantillon liquide sont retenus par la phase solide alors que le solvant constituant l'échantillon est éliminé, les analytes sont ensuite élués avec un petit volume d'un solvant de forte force éluante (3).

De nombreux supports d'extraction et de purification ont été developpés et sont actuellement disponibles sous divers formats (2). Cependant, ces supports mettent principalement en œuvre des interactions de nature hydrophobe entrainant alors à la co-extraction d'un grand nombre de composés de polarité similaire à celle de l'analyte recherché et rendant ainsi l'analyse chromatographique difficile d'un point de vue quantitatif.

Le developpement d'immunadsorbants (IS) a permis de pallier ce manque de sélectivité en exploitant l'intéraction antigène-anticorps. En effet, les anticorps développés contre l'analyte cible sont gréffés sur un support solide, la sélectivité est alors obtenue grâce au mécanisme de reconnaissance moléculaire mis en jeu. Même si le potentiel de ces supports a été largement démontré (4), le coût et la durée de developpement des are non covalent like - hydrogen bondings, $\pi$ - $\pi$ or electrostatic interactions. The most common approach to the synthesis is the bulk polymerisation to obtain a monolithic polymer: MIPS for SPE can be conditioned in cartridges or directly integrated in the analytical system. Many applications have already highlighted the high potential of MIPs for the selective extraction of target compounds from biological samples.

\section{KEY-WORDS}

Molecularly imprinted polymers, Solid-phase extraction, Selectivity, Biological samples.

IS ont conduit à l'utilisation d'un support synthétique appelé polymères à empreintes moléculaires (MIPs).

Au même titre que les anticorps ou les enzymes, les polymères à empreintes moléculaires sont des réseaux de polymères réticulés possèdant des sites récepteurs spécifiques d'une molécule cible. Les MIPs sont utilisés dans divers domaines comme la catalyse, la séparation chirale, les biocapteurs...(5). L'utilisation des MIPs en tant que support d'extraction est actuellement en plein developpment. La première application a été publiée en 1994 par B. Sellergren pour l'extraction de la pentamidine présent à l'état de traces dans l'urine (6). Environ une cinquantaine d'applications ont été publiées depuis pour l'extraction et la purification sélective de composés présents dans des échantillons biologiques comme en témoigne le tableau I (6-51). Des micropolluants organiques ont été extraits par ces supports à partir de matrices biologiques tel que le bisphénol A du sérum et des pesticides (triazines) du foie et de l'urine. Beaucoup de MIP ont été synthétisés pour l'extraction du clenbutérol (dopant) à partir du foie et de l'urine. La quercétine (flavonö̈de) ainsi que le cholestérol ont été extraits du plasma. Des alcaloïdes comme la caféine, la nicotine, la théophylline, la sinoménine, la scopalamine, l'harmaline et de nombreux autres composés d'intérêt pharmaceutique (ibuprofène, darifénacine, bupivacaïne, metformine, vérapamile, propranolol, phenytoïne, tamoxifène, phenobarbital, diazépam...) en particulier des antibiotiques (céphalexine, chloramphenicole, tylosine, ciprofloxacine, oxytétracycline, triméthoprime), et un neurotransmetteur (dopamine) ont été extraits à partir de sérum, plasma ou encore de l'urine. Enfin, quelques applications ont été réalisées très récemment sur l'extraction de métaux à partir de supports imprimés (52). Toutes ces applications témoignent ainsi du large spectre d'utilisation actuel de ces supports en tant que phase SPE pour les milieux biologiques $(53,54)$. 


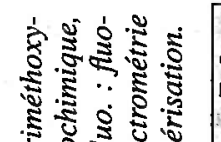
(2)

है

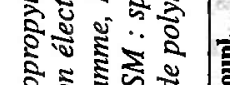

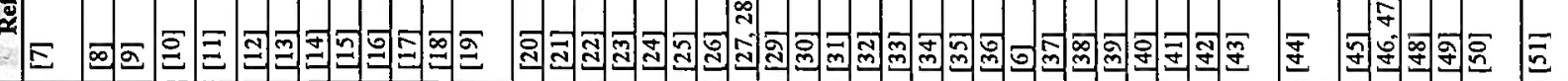

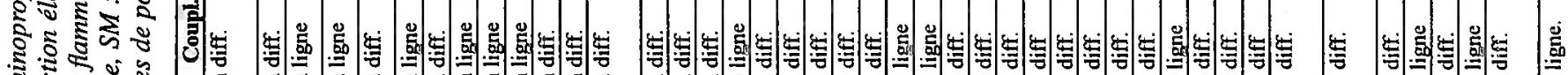

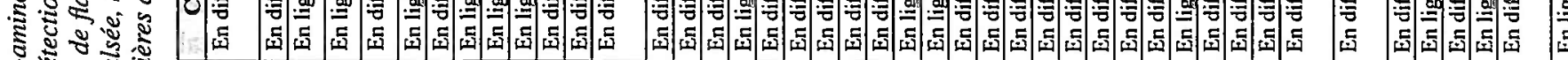

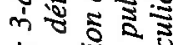

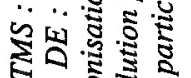

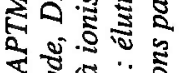

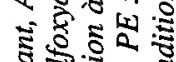

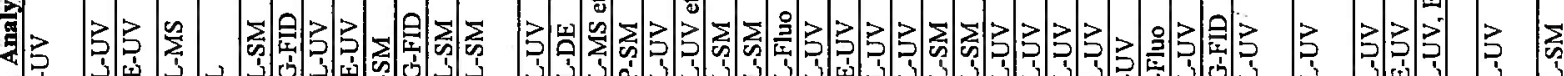

络

:

उี

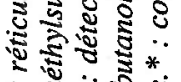

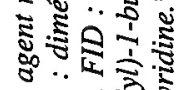

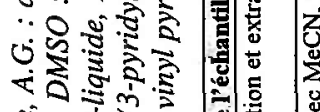

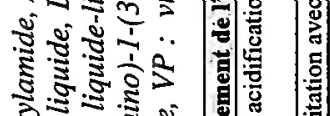

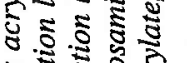

约

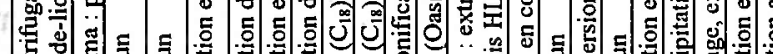

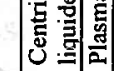

言

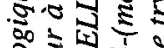

ษ

ำ

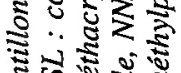

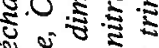

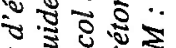

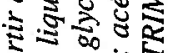

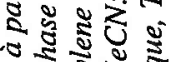

.

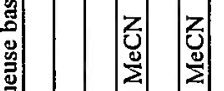

巽

:

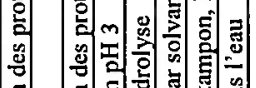

5.

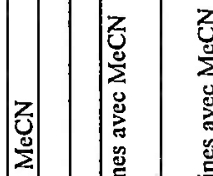

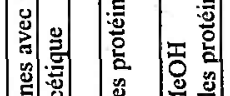

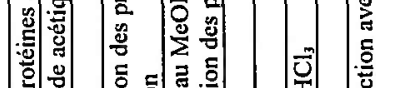

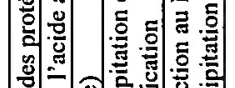

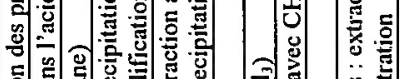

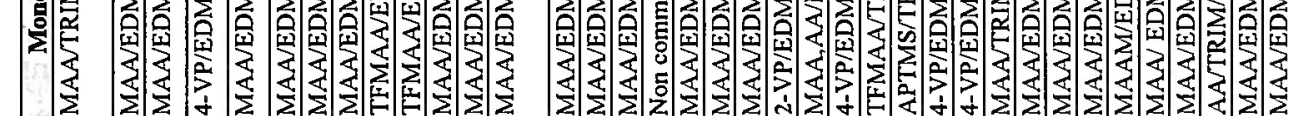

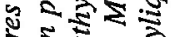

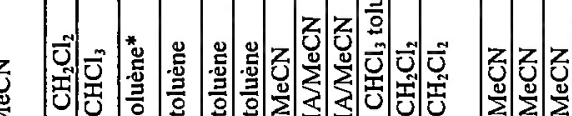

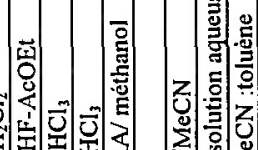

ty-

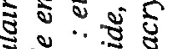

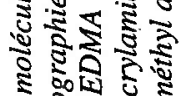

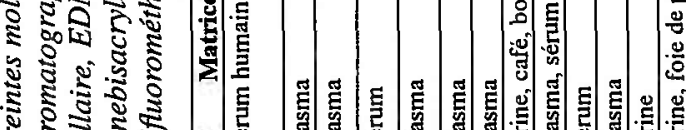

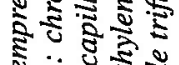

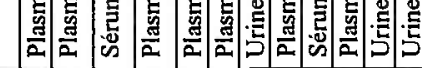

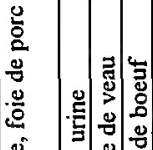

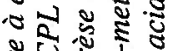

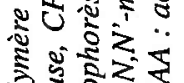

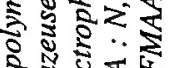

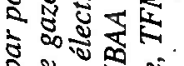

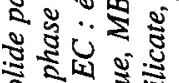

कำ

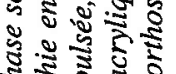

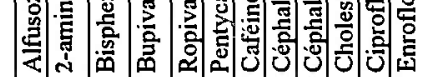

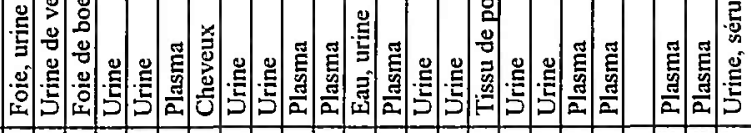

产

졸

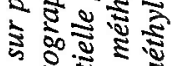

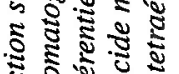

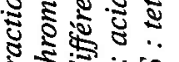

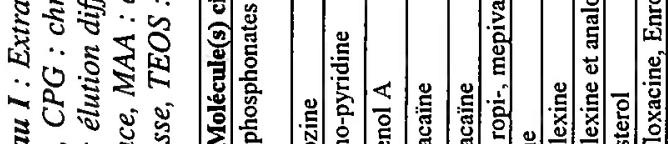

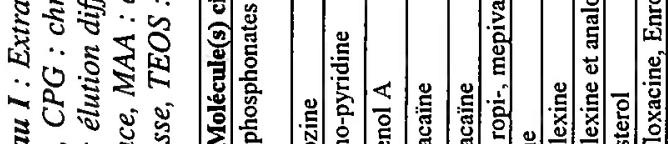

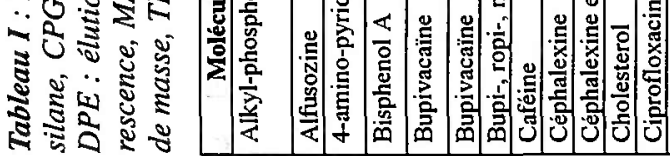

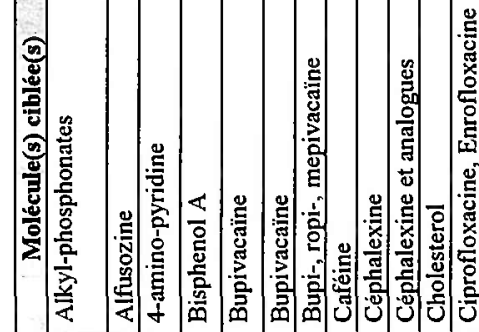

\section{产哭}

$\overline{0}$

$\overline{0}$

密

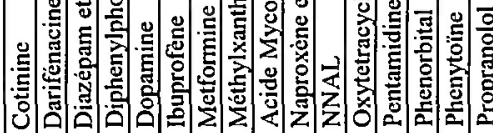

:

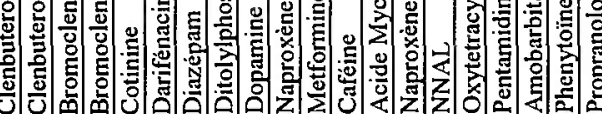

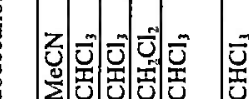
(O)

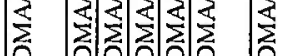




\section{Synthèse de MIP pour la SPE}

\section{Principe de l'impression moléculaire}

L'impression moléculaire consiste à créer des images complémentaires en termes de structure et de fonctionnalités chimiques d'une molécule cible au sein d'un polymère synthétique. Cette étape est cruciale car elle conditionne la sélectivité du MIP résultant et donc ses performances. Le principe de la synthèse d'un MIP est illustré figure 1. Pour ce faire, une molécule modèle, nommée molécule empreinte ou template est mise en présence, dans le solvant de synthèse, de monomères choisis pour leur grande affinité avec la molécule empreinte, d'un agent réticulant et d'un initiateur de polymérisation. Par initiation thermique ou photochimique, les monomères polymérisent autour de la molécule empreinte ce qui induit la création de cavités spécifiques présentant une complémentarité structurale avec la molécule empreinte. La présence d'un solvant porogène permet le développement d'une structure macroporeuse. Une fois la polymérisation achevée, la molécule empreinte est éliminée de la matrice polymérique ce qui conduit à la formation d'un polymère rigide renfermant des sites de reconnaissance spécifique de la molécule modèle.

La sélectivité résultante du polymère dépend en fait de l'affinité de la molécule empreinte vis-à-vis des monomères et par conséquent, du nombre et de l'intensité des interactions initiées entre les deux entités. Des interactions de nature non covalente, covalente, ou de type métal-ligand peuvent être développées. L'approche la plus courante pour leur utilisation en extraction sur phase solide est la technique d'impression non covalente (55). Dans ce procédé, le complexe entre la molécule empreinte et le monomère est formé in-situ par des interactions non covalentes de type liaisons hydrogène, intéractions $\pi-\pi$ ou liaisons électrostatiques. Pour une sélectivité optimale lors de l'extraction, les mêmes interactions doivent se developper entre l'analyte et le MIP. Cette méthode est très facile à mettre en oeuvre, l'extraction de la molécule empreinte de la matrice polymérique est également aisée. Cependant, l'obtention d'un complexe stable lors de la synthèse conditionne le choix du monomère et restreint le choix du solvant de polymérisation, celui-ci devant solubiliser les différents réactifs tout en stabilisant le pré-complexe, doit être peu polaire et aprotique.

Une seule application a été réalisée à ce jour avec un MIP synthétisé par une approche covalente pour l'extraction de composés organiques à base d'étain dans des tissus de moule (56). L'approche semi-covalente a été abordée en combinant lors de la synthèse, le développement de liaisons covalentes et non covalentes, en vue de l'extraction de composés phénoliques à partir d'eaux de rivières mais les auteurs ont mentionné un apport en sélectivité faible par rapport au MIP synthétisé par approche non covalente avec la même molécule empreinte (57).

\section{Choix des réactifs}

Dans une approche non covalente, le solvant de polymérisation est un des facteurs clé pour obtenir une impression moléculaire optimale car la stabilité du complexe formé lors de la polymérisation dépend de ses paramètres physico-chimiques (58). Pour favoriser le developpement d'interactions non covalentes comme des laisons hydrogène, un solvant peu polaire et aprotique est généralement utilisé tel que le dichlorométhane, le chloroforme ou encore le toluène. Des synthèses peuvent également être réalisées dans l'acétonitrile. Même si la majeure partie des MIP est synthétisée en milieu organique pour des contraintes de solubilisation de l'ensemble des réactifs, des solvants polaires protiques ont été utilisés comme l'eau et le

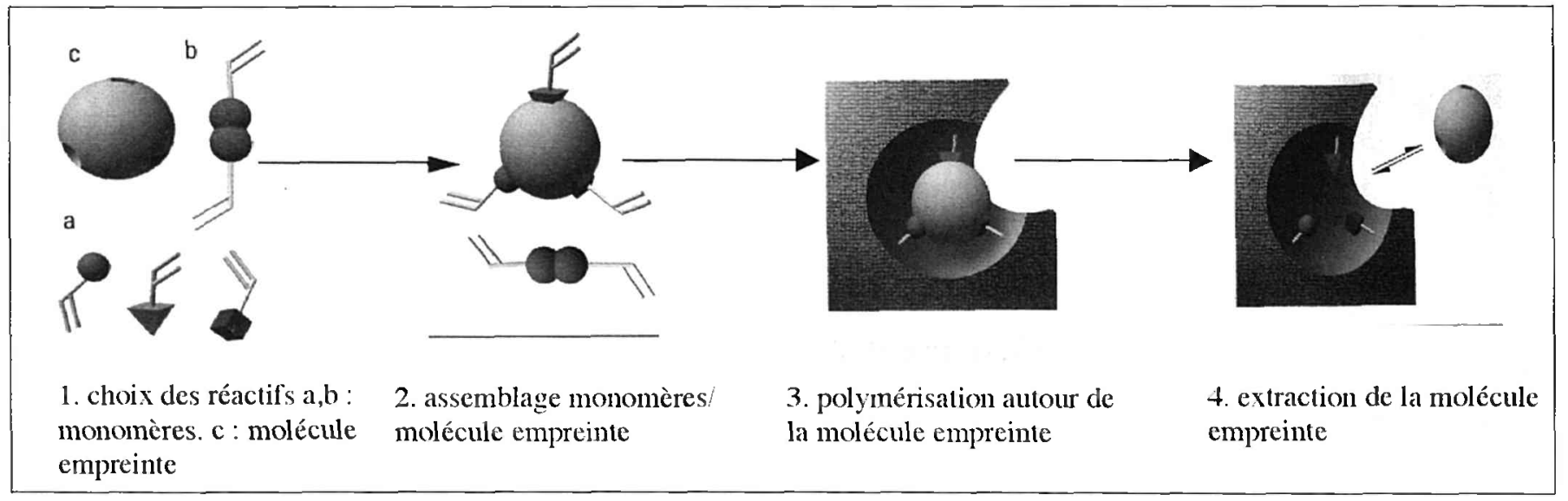

Figure 1 : Principe de synthèse d'un polymère à empreintes moléculaires. Adapté de (80) avec la permission de ACS. 
méthanol mais pour des applications environnementales $(59,60)$. Seule une synthèse en milieux aqueux a été récemment proposée pour l'extraction de méthylxanthines à partir d'urine en utilisant des organosilanes comme monomères (32) contrairement aux autres travaux qui ont tous été basés sur l'utilisation de monomères organiques.

Concernant le choix de la molécule empreinte, un problème intrinsèque, dû au mode de synthèse, est le risque d'une libération tardive de la molécule empreinte à partir de la matrice polymérique lors de l'utilisation du support. Ce phénomène provient du fait que l'élimination de la molécule empreinte après polymérisation est parfois difficile. Cela peut être gênant lors de la quantification de l'analyte cible après extraction car le résultat peut être erroné par une surestimation de la quantité de molécule cible détectée. Pour éviter ce problème, beaucoup de travaux sont à présent réalisés en utilisant un analogue du composé ciblé comme molécule empreinte $(9,10,12,13,22,23,30,37,43)$.

Le choix du monomère a également une grande importance sur la sélectivité résultante du MIP. Le monomère idéal doit développer de fortes interactions avec la molécule empreinte, être stable et évidemment être soluble dans le solvant de polymérisation. Malgré la grande variété de monomères disponibles, la majorité des MIP utilisée en SPE est synthétisée avec l'acide méthacrylique (MAA) car il a la faculté d'être donneur et accepteur de liaisons hydrogène. De plus, par ses propriétés acides, il peut développer des interactions électrostatiques avec des molécules empreintes basiques. Ainsi, le MAA est le monomère choisi quasiment par défaut dès que les molécules cibles sont neutres ou basiques. A l'inverse, pour des molécules acides, on cherchera un monomère basique capable également de développer des liaisons hydrogène et éventuellement des interactions électrostatiques. Pour ces raisons, le 4-vinylpyridine (4-VP) et éventuellement le 2-VP sont les monomères basiques les plus utilisés pour des MIP dédiés à la SPE. Les agents réticulants les plus couramment utilisés sont le diméthacrylate d'éthylène glycol (EDMA) et le triméthylpropane tryméthacrylate (TRIM). Enfin, l'initiateur le plus courant est le 2,2'-azobisobutyronitrile (AIBN).

Des approches combinatoires ont été développées afin de sélectionner au mieux le monomère sans se cantonner au choix limité du MAA ou du 4-VP. Le principe repose sur l'évaluation d'un grand nombre de MIP synthétisés dans des conditions expérimentales différentes (nature et/ou teneur en monomères). A ce titre, le groupe de B. Sellergren a proposé une méthode semi-automatisée pour la synthèse et l'évaluation de MIPs à hauts débits : 80 MIPs ont été synthétisés et évalués en termes de rétention et de sélectivité en une semaine seulement. Cette méthode a été appliquée pour optimiser la synthèse de MIPs pour la reconnaissance de la phenytoïne, la nifédine et la bupivacaïne $(11,61)$. Une autre approche a été abordée par le groupe de $S$. Piletsky en utilisant la modélisation moléculaire. Le principe est de calculer les énergies d'interaction développées entre la molécule empreinte et les différents monomères appartenant à une bibliothèque virtuelle et de sélectionner les monomères aboutissant à un complexe monomère-molécule empreinte le plus stable possible. Des MIPs microcystine-LR (62), tylosine (63) et abacavir (64) ont déjà été synthétisés avec des monomères autres que l'acide méthacrylique et choisis au moyen de cette modélisation. Récemment, une approche similaire mais simplifiée a été utilisée pour le choix du solvant optimal pour la synthèse d'un MIP nicotinamide (65). L'utilisation de données RMN conjuguées avec une approche par modélisation moléculaire a permis également d'optimiser le ratio molécule empreinte/monomère ainsi que le choix du solvant (66). Enfin, l'étude des spectres UV a été utilisée pour l'évaluation des différents complexes ainsi que pour le choix du monomère (67) et du ratio molécule empreinte/monomère (68).

\section{Méthodes de polymérisation}

Différents modes de polymérisation existent pour la conception d'un polymère à empreintes moléculaires. Ainsi, suivant la synthèse, le MIP résultant peut se présenter sous la forme d'un monolithe ou de particules sphériques poly- ou mono-disperses (55).

La polymérisation en bloc est la plus populaire dans le domaine de la SPE de par sa facilité de mise en œuvre. Elle est également la plus ancienne. Dans un premier temps, tous les réactifs sont mis en présence dans un tube à sceller. Un dégazage sous diazote est nécessaire de manière à éliminer le dioxygène dissous en solution qui favoriserait la production de radicaux libres (61). Le tube est ensuite placé dans un bain thermostaté (0$15^{\circ} \mathrm{C}$ ) à proximité d'une lampe à rayonnement ultraviolet si la polymérisation est initiée de façon photochimique ou dans un bain thermostaté à $60^{\circ} \mathrm{C}$ pour une initiation thermique. La polymérisation dure environ $24 \mathrm{~h}$ et aboutit à un monolithe. Le tube est alors brisé. Le matériau est ensuite broyé puis tamisé de manière à obtenir au final des particules de granulométrie comprises généralement entre 25 et $36 \mu \mathrm{m}$. Une étape de sédimentation est alors nécessaire afin d'éliminer les plus fines particules $(<25 \mu \mathrm{m})$. Le MIP est ensuite conditionné en cartouche ou en précolonne. L'étape d'extraction de la molécule empreinte peut-être réalisée soit par extraction en solvant chaud juste avant l'étape de broyage ou par des percolations successives 
de solvants une fois le MIP prêt à l'emploi. Malgré sa simplicité, ce mode de synthèse reste relativement long et fastidieux. De plus, une grande fraction de polymères est perdue sous forme de fines particules. Enfin, les particules obtenues ne sont pas forcément sphériques et de taille homogène ce qui peut-être gênant pour certaines applications autres que la SPE. D'autres approches ont été envisagées pour l'obtention de particules sphériques mais de façon très anecdotique dans le domaine de la SPE telles que la polymérisation par suspension, précipitation, multi-étapes, core-shell (69). L'approche par synthèse in-situ commence à se développer en vue de l'utilisation des MIPs dans des systèmes miniaturisés (70).

\section{Caractérisation des sites de reconnaissance}

La nature non covalente des interactions développées lors de la formation du complexe monomère-molécule empreinte conduit à un support possédant des cavités de structure plus ou moins bien définies qui doivent être mises à profit lors de l'étape d'extraction. Parallèlement, le polymère peut retenir les composés en dehors des cavités. Ces interactions développées, non spécifiques, sont alors de plus faible énergie que celle stabilisées par complémentarité stérique au sein des cavités. Pour quantifier ces interactions non spécifiques, un polymère non imprimé (NIP) doit être réalisé en parallèle de la synthèse du MIP, le procédé de synthèse étant identique à celui du MIP à l'exception de la molécule empreinte qui n'est pas introduite dans le milieu réactionnel.

Une des méthodes les plus courantes pour évaluer alors la spécificité d'un MIP est le tracé d'isothermes d'adsorption. Cette approche repose sur l'établissement d'un équilibre régissant la formation du complexe molécule cible-MIP lors de l'extraction. Ces isothermes sont faites en solution en présence de la molécule cible et du MIP ou du NIP afin que l'équilibre soit atteint. La figure 2 montre le tracé de deux isothermes d'adsorption de la cotinine obtenu sur le MIP et sur le NIP dans l'acétonitrile. L'obtention de palier à fortes concentrations traduit la saturation des sites : la différence de concentration fixée d'analyte entre les deux paliers (MIP et NIP) traduit le nombre de cavités spécifiques. Ainsi, la comparaison des deux isothermes indique une plus forte capacité de rétention pour le MIP que pour le NIP. Une analyse Scatchard permet ensuite d'estimer les constantes de formation du complexe afin d'évaluer l'affinité du MIP vis-à-vis de la molécule ciblée (17).
La sélectivité du MIP peut être également caractérisée par chromatographie en phase liquide. Cette approche consiste à introduire le support imprimé et non imprimé dans des colonnes, les supports sont alors utilisés comme phase stationnaire dans le système chromatographique. La plupart des études portent sur des MIP en bloc comportant une très grande hétérogénéité en terme de granulométrie induisant des pics d'élution chromatographiques relativement larges. Néanmoins, cette approche permet d'estimer les facteurs de rétention de chaque support : le rapport de ces deux grandeurs, définissant le facteur d'impression IF (Imprinting Factor), est très informatif sur la sélectivité potentiel du MIP $(18,19,33,71-73)$.

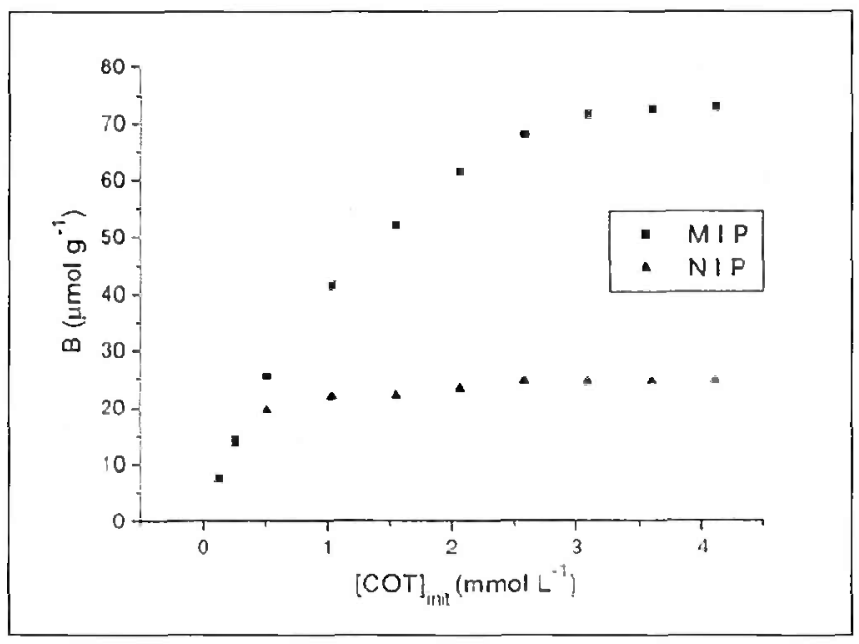

Figure 2 : Isothermes d'adsorption de la cotinine sur le MIP et sur NIP : quantité de cotinine extraite par gramme de support en fonction de la concentration initiale présente dans la solution d'acétonitrile. De (24) avec la permission de Springer-Verlag.

\section{Extraction sélective sur MIP}

Le format le plus utilisé pour l'extraction sur MIP reste la cartouche (15-500 $\mathrm{mg}$ de phase) pour une utilisation en différé. Le principe de mise en œuvre d'une extraction sur MIP est similaire à celui d'une extraction avec un support conventionnel (3), il est illustré figure 3. La première étape consiste à conditionner le support contenu dans la cartouche d'extraction. Lors de la seconde étape, on procède à la percolation de l'échantillon sur le support. Selon la nature de l'échantillon biologique (urine, plasma...), des composés à analyser et le facteur d'enrichissement recherché, le volume d'échantillon percolé peut varier de quelques microlitres à quelques dizaines de millilitres. Une étape de 
lavage (étape 3) doit permettre de rompre les interactions non spécifiques et d'éliminer ainsi les composés interférents faiblement retenus sur le support. Enfin, on procède à l'élution des composés ciblés en faisant percoler un solvant spécifiquement choisi pour rompre les interactions mises en jeu entre les analytes d'intérêt et le support solide. L'éluat est ensuite généralement évaporé puis repris dans un solvant compatible avec le système chromatographique avant d'être analysé.

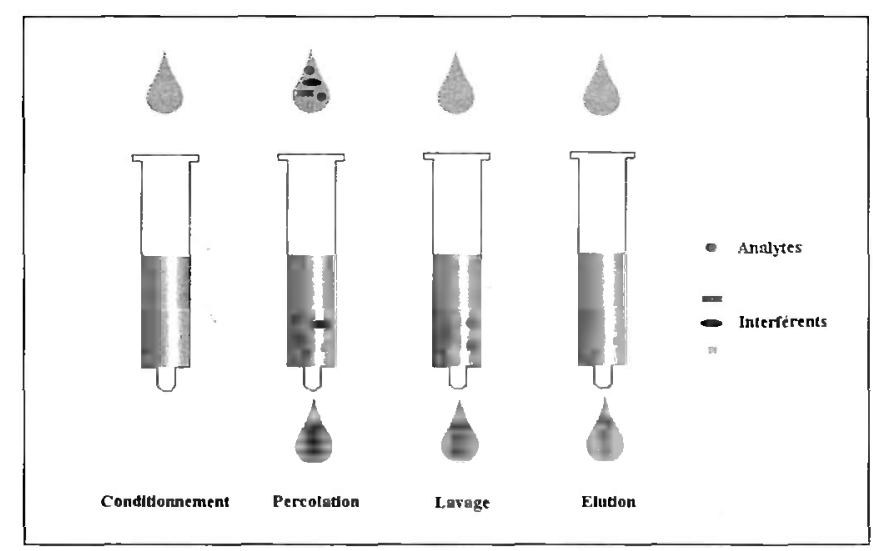

Figure 3 : Principe de l'extraction sur phase solide. Adapté d'un document Gilson.

Le développement d'une procédure d'extraction sélective implique de connaitre les mécanismes d'interactions développés entre le support et l'analyte cible. Ainsi, les solvants de lavage et d'élution constituent les paramètres clés de l'extraction pour l'obtention d'une sélectivité optimale car ils régissent les interactions entre le composé cible et le MIP.

Une reconnaissance sélective est obtenue dans un solvant proche du milieu de synthèse afin de développer des interactions MIP-molécule empreinte au sein des cavités similaires à celles développées lors de la formation du complexe monomère-molécule empreinte avant polymérisation (58). Concernant la nature des solvants de polymérisation, le tableau I met clairement en évidence l'utilisation systématique de solvants organiques aprotiques tels que le dichlorométhane, le chloroforme, le toluène ou encore l'acétonitrile. Par conséquent, les propriétés de reconnaissance moléculaire du MIP seront exploitées de façon optimale en milieu peu polaire et aprotique favorisant le développement d'interactions hydrophiles telles que des liaisons hydrogène et éventuellement des interactions électrostatiques. Ainsi, la procédure d'extraction sélective consistera à percoler les analytes dans un solvant peu polaire et aprotique afin qu'ils développent des interactions spécifiques au sein des cavités du MIP. Une étape de lavage réalisée avec un solvant de même nature permet d'éliminer les éventuels interférents faiblement retenus sur le polymère. L'optimisation de cette étape est cruciale pour aboutir à une sélectivité optimale mais également très délicate car les interactions analyte-polymère sont plus ou moins fortes en fonction de la nature de l'analyte et de la structure des cavités. La réelle optimisation de cette étape ne peut se faire qu'avec l'utilisation en parallèle du NIP $(8,17,24)$ en assurant l'élution des composés lors de cette étape sur le NIP et leur rétention sur le MIP. De façon générale, des solvants organiques de faible polarité sont utilisés avec un faible pourcentage de solvant polaire. La figure 4 présente les profils d'élution obtenus sur un MIP alfuzosine et un NIP synthétisé dans du dichlorométhane avec de l'acide méthacrylique comme monomère (8). La percolation de l'alfuzosine a été réalisée dans le milieu de synthèse à savoir le dichlorométhane. Afin, de rendre le lavage sélectif, $2 \%$ de méthanol ont du être ajoutés au dichlorométhane ce qui a permis d'éluer l'alfuzosine sur le NIP par rupture des liaisons hydrogène non spécifiques tout en retenant le produit sur le MIP par une rétention uniquement liée à la présence de cavités spécifiques. L'élution a ensuite été assurée par percolation du méthanol compte tenu de la nature polaire des interactions mises en jeu dans ce cas. $93 \%$ de rendement d'extraction ont été obtenus sur le MIP avec seulement $2 \%$ sur le NIP ce qui témoigne de la grande sélectivité du protocole.

Une fois le protocole sélectif mis en place, il peut également être exploité pour la détermination de la capacité du support à savoir le nombre de sites spécifiques que possèdent le MIP. Pour ce faire, des quantités croissantes d'analyte sont percolés sur le support, la capacité est alors atteinte lorsque le rendement d'extraction n'est plus constant et chute $(8,74)$. D'une manière générale, la capacité d'un MIP est comprise entre $1-40 \mu \mathrm{mol} / \mathrm{g}(75,76)$.

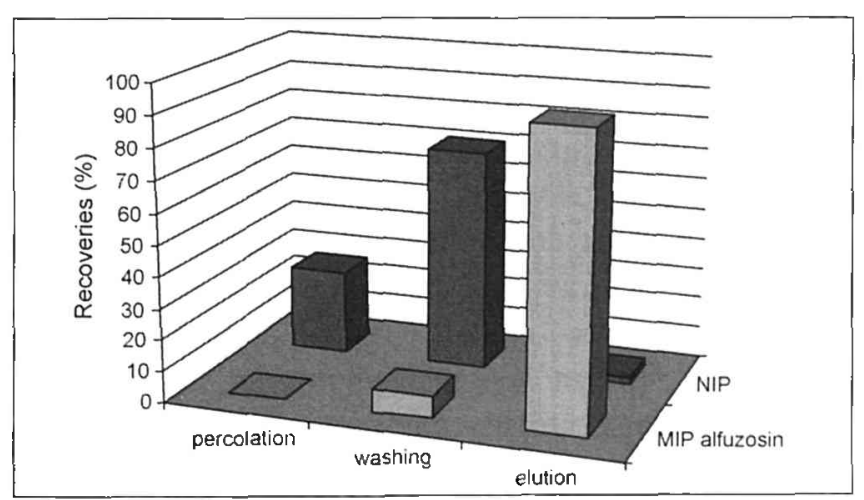

Figure 4: Profils d'élution de l'alfizosine obtenus sur MIP et sur NIP après la percolation de $500 \mu \mathrm{L}$ de dichlorométhane dopé avec $200 \mathrm{ng}$ d'alfuzosine. Adapté de (8) avec la permission d'Elsevier: 


\section{Applications des MIPs aux échantillons réels}

Pour la purification d'échantillons biologiques solides, les extraits sont généralement dissous dans un solvant de nature très proche du solvant de polymérisation $(20$, $26,36,48)$ ce qui permet d'obtenir une grande sélectivité dans l'extraction. Très récemment, un MIP diazepam a été synthétisé dans du chloroforme avec de l'acide méthacrylique comme monomère et utilisé pour l'extraction de cette benzodiazépine à partir de cheveux : 93\% de rendement d'extraction ont été obtenus sur le MIP avec seulement $16 \%$ sur le NIP (26). La procédure a également permis d'extraire d'autres benzodiazépines mais avec une sélectivité moindre. Les performances du MIP en terme de sélectivité ont également été démontrées par comparaison avec un support classique. Pour illustration, un MIP développé pour l'extraction sélective du cholestérol a été appliqué pour la purification d'un extrait de jaune d'œuf préalablement traité par saponification puis extrait à l'hexane (17). L'extrait traité a également été percolé sur un support $\mathrm{C}_{18}$. La figure 5 présente les chromatogrammes obtenus pour l'analyse du cholestérol à partir de jaune d'œuf sans procédure de purification et avec extraction sur $\mathrm{C}_{18}$ ou MIP ce qui témoigne de l'intérêt des MIPs pour le traitement de matrices complexes.

Concernant les échantillons biologiques liquides (plasma, sérum, urine...) une première étape d'extraction conventionnelle par extraction liquide-liquide $(7,17$, $20,42,46,47)$ ou par extraction sur phase solide sur des supports conventionnels type $C_{18}(15,16,18,19$, 49) peut avoir lieu afin de transférer l'analyte dans un milieu organique mieux adapté à la percolation sur MIP surtout si des interactions sélectives polaires sont attendues. Cependant, beaucoup d'applications se font à présent par percolation directe de l'échantillon biologique sur le MIP. La procédure d'extraction est tout d'abord élaborée en milieu aqueux pure ou tamponné avec un contrôle sur le NIP.

La percolation directe d'échantillons aqueux sur le MIP induit généralement une rétention des composés par interactions hydrophobes non spécifiques, le développement d'interactions spécifiques au sein des cavités se faisant alors lors de l'étape de lavage par percolation d'un solvant adapté. Néanmoins, certains solvants de lavage utilisés peuvent être non miscibles comme le dichlorométhane, le support doit alors être séché à l'azote entre les deux étapes ou la procédure doit être adaptée en ajoutant des étapes de lavage intermédiaires par des solvants miscibles. En outre, les propriétés acido-basiques des monomères utilisés peuvent

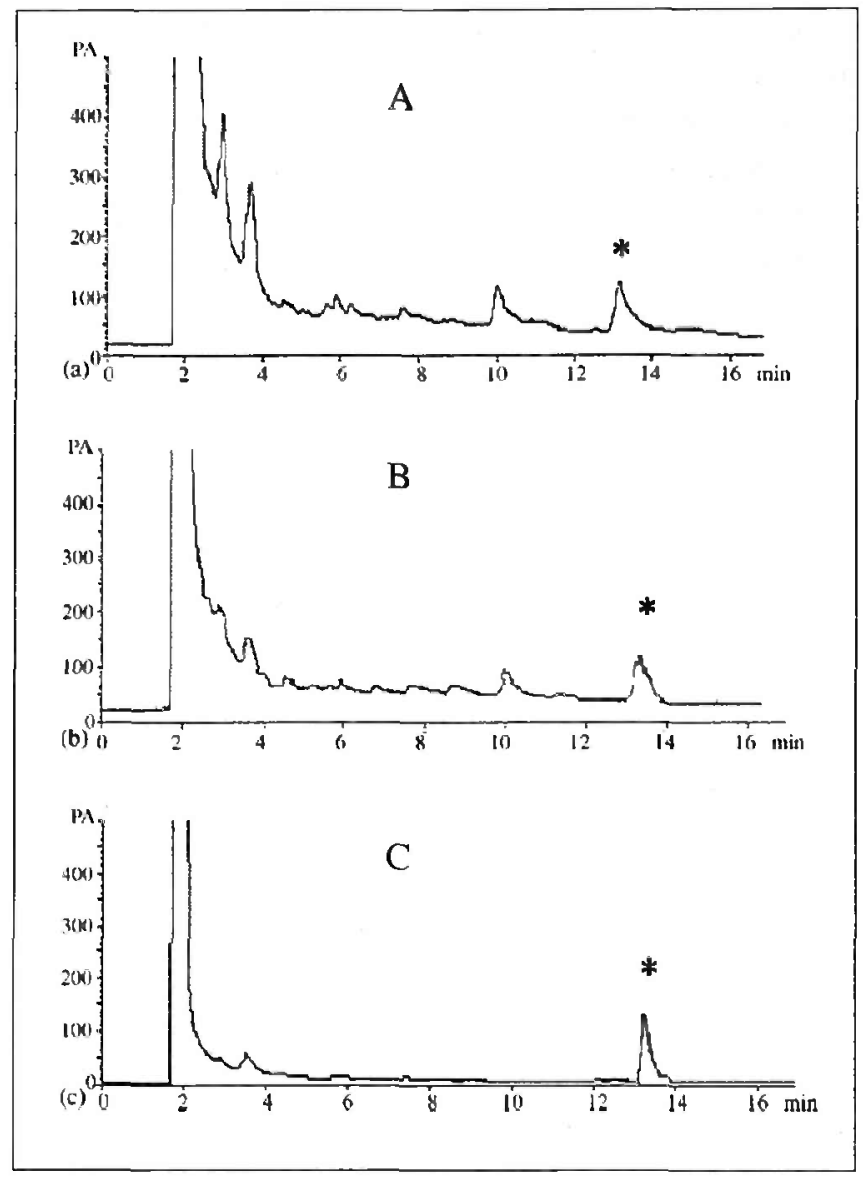

Figure 5: Chromatogrammes résultant de l'injection de l'extrait traité de jaune d'cuf avant $(A)$ et après extraction sur $C_{18}(B)$ et sur MIP $(C)$. Extrait dopé à $50 \mu \mathrm{g} / \mathrm{mL}$. Adapté de (I7) avec la permission d'Elsevier.

engendrer le développement d'interactions électrostatiques entre le composé cible et le support. A titre d'exemple, la cotinine, le principal métabolite de la nicotine a pu être extraite sélectivement et directement de l'urine à partir d'un MIP synthétisé dans du dichlorométhane avec de l'acide méthacrylique grâce à un lavage en milieu alcalin qui a permis de masquer les fonctions acides du monomère qui engendrait une rétention non spécifique trop importante (24). Pour illustration, la figure 6 compare le chromatogramme résultant d'une injection directe d'urine de fumeur par rapport au chromatogramme résultant de l'extraction au préalable sur le MIP cotinine. L'apport en sélectivité par le MIP est largement mis en évidence. En effet, un grand nombre d'interférents est éliminé par le MIP ce qui rend l'identification et la quantification du composé cible très aisée. L'apport en sélectivité peut également être démontré en dopant dans l'échantillon des composés interférents de structure et de polarité similaires, leur absence de rétention sur le support confirme alors la présence de cavités spécifiques de la molécule cible (77). Enfin, de nombreuses études ont également 
mis en évidence l'existence d'une zone de $\mathrm{pH}$ optimal pour l'extraction correspondant à l'ionisation des deux espèces impliquées (analytes et résidus de monomères) lorsque la rétention est basée sur le développement d'interactions électrostatiques $(13,27,66,78)$.

L'application des MIPs aux échantillons réels induit parfois une baisse des rendements d'extraction par la présence de composés interférents comme les protéines ou les sels. Ainsi, les protéines contenus dans le plasma ou le sérum sont généralement préalablement précipitées par ajout d'un solvant organique ou d'acide ( 7 , $8,25,31,33,40,44,79)$. Les effets de matrice notamment induits par la présence de composés ioniques peuvent aussi être atténués par simple dilution de l'échantillon ou par modification du $\mathrm{pH}$ afin de diminuer la contribution des interactions électrostatiques (11-13, $28,29,41,50,73$ ).

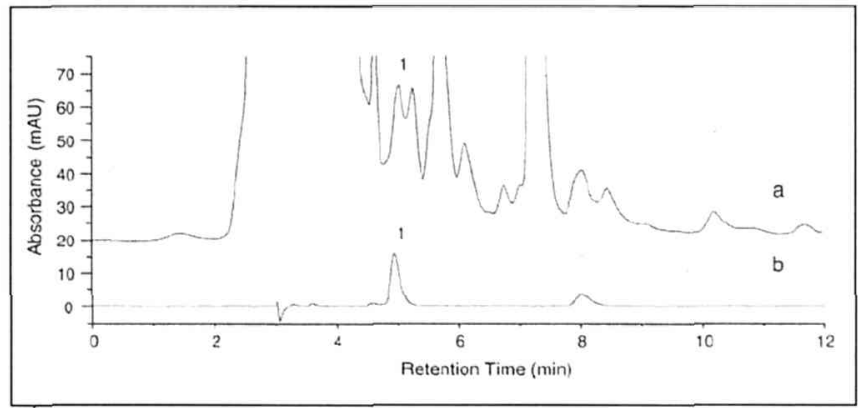

Figure 6 : Chromatogrammes obtenus après injection d'une urine de fumeur avant $(A)$ et après extraction sur $M I P(B)$. Longueur d'onde de détection : $260 \mathrm{~nm}$. (1) : cotinine. Adapté de (24) avec la permission de Springer-Verlag.

\section{Couplage en ligne}

Le principal avantage de l'extraction en différé est la facilité de mise en ouvre : la procédure est simple et aucune contrainte n'est imposée sur le choix des solvants d'élution car ils n'ont pas d'influence sur la qualité de l'analyse chromatographique. Néanmoins, ce mode d'extraction ne permet pas d'intégrer totalement l'étape de traitement de l'échantillon au système analytique. A cet effet, des procédés d'extraction sélective sur MIP couplée en ligne avec la chromatographie en phase liquide ont été développés.

Le principe du couplage en ligne est illustré figure 7. Le MIP est introduit dans une précolonne installée sur une vanne de commutation à six voies sur laquelle sont également connectées une pompe de préconcentration, une pompe analytique et la colonne analytique. Après le conditionnement du MIP, l'échantillon est percolé grâce à la pompe de préconcentration. Les analytes sont alors piégés sur le support. Une étape de lavage

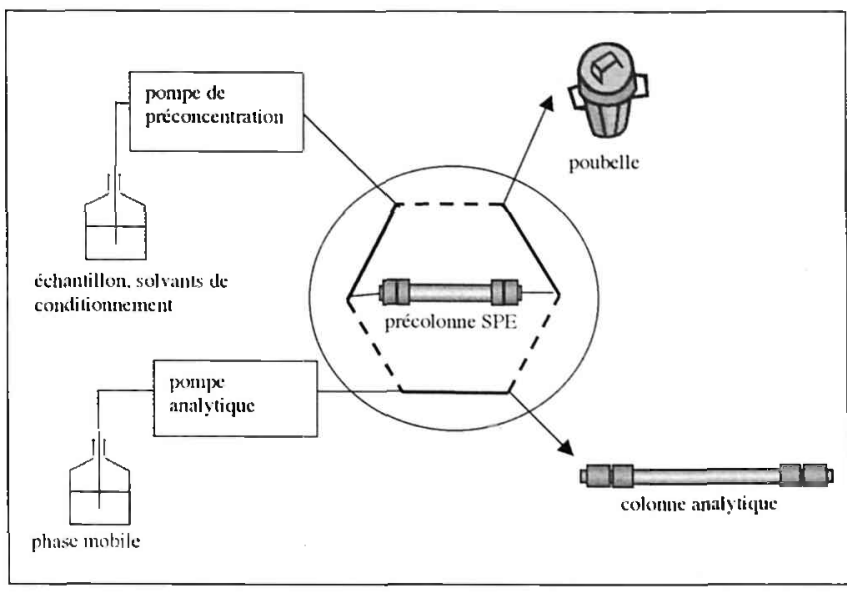

Figure 7 : Couplage en ligne de l'extraction sur phase solide avec la chromatographie en phase liquide.

peut être introduite si nécessaire. L'étape d'élution est ensuite assurée par la phase mobile de la séparation chromatographique : par commutation de la vanne, la phase mobile passe alors sur la précolonne pour éluer les composés et les entraîner vers la colonne analytique afin qu'ils soient séparés. Ce couplage en ligne permet à ce que la totalité des composés extraits sur le support soit analysée. Ainsi, le gain en sensibilité obtenu permet de réduire le volume des échantillons. Cependant, très peu d'applications couplant en ligne directement le MIP à la colonne analytique ont été jusqu'à maintenant réalisées $(10,14,30,39)$. Dans ce type de couplage, la difficulté réside dans la compatibilité des solvants utilisés lors de l'extraction avec l'analyse chromatographique. Le transfert du MIP vers la colonne analytique doit être suffisamment rapide pour ne pas occasionner de perte en efficacité lors de la séparation chromatographique. Ainsi, une réelle optimisation de la procédure d'extraction doit être réalisée à nouveau par rapport à une procédure éventuellement déjà élaborée en différé pour assurer de hauts rendements d'extraction tout en gardant un degré de sélectivité élevé. L'extraction en ligne sur MIP peut également être couplée à une autre extraction sur phase solide $\left(\mathrm{C}_{18}\right)$ réalisée en amont $(15,16,49)$. Un support de type RAM (phase à accès restreint) a été également utilisé avant le support MIP pour l'extraction du vérapamile à partir de l'urine (51). Cette approche donne des résultats intéressants en terme de rendements d'extaction et de sélectivité mais elle nécessite cependant l'optimisation de deux extractions en ligne et peut-être longue à réaliser.

\section{Conclusions et perspectives}

En quelques années, le potentiel des MIPs a largement été démontré pour leur utilisation en extraction sur phase solide pour divers échantillons biologiques. Leur simplicité de fabrication et leur faible coût de synthèse 
font des MIPs un support de plus en plus attrayant. Pour preuve, des sociétés (MIP technologies, PolyIntell...) propose dèjà la synthèse de MIPs à façon. Dans le domaine académique, de nombreux groupes de recherche font maintenant leurs propres MIPs en vue du developpement de nouvelles extractions sélectives de diverses molécules d'intérets. Néanmoins, il faut être conscient que l'apport de la sélectivité par ce support peut être limité par le mode de synthèse actuellement utilisé. En effet, la majeure partie des MIPs est synthétisée par polymérisation en bloc en milieu organique avec des monomères vinyl ou acrylique ce qui induit une rétention par liaisons hydrogène ou intéractions électrostatiques. L'utilisation de ces supports directement en milieu aqueux induit donc généralement une rétention non spécifique que seule une étape de lavage par un solvant approprié peut rendre sélective. Ainsi, le developement d'une procédure d'extraction sélective doit passer par la connaissance approfondie du mécanisme de rétention afin d'optimiser au mieux la procédure, le MIP n'étant pas intrinsèquement sélectif. Le contrôle sur le NIP est également capital pour s'assurer de l'apport réel en sélectivité durant l'extraction.

De nombreux developpements doivent encore avoir lieu notamment au niveau du mode de synthèse. A l'heure actuelle, les molécules empreintes de polarité modérée et présentant un caractère acido-basique marqué sont les mieux adaptées à la synthèse de MIPs générallemnt effectuées en milieu organique. Ainsi, la synthèse de MIPs pour des molécules polaires non solubles dans ces milieux organiques pose encore problème. Il existe également une importante demande au niveau de l'extraction sélective de protéines et plus largement pour les macromolécules polaires. Cependant, grâce à leur grande capacité et leur haut degré de sélectivité, déjà démontré pour un grand nombre de molécules, l'utilisation des MIPs dans des systèmes miniaturisés via une polymérisation in-situ est un domaine extrêmement prometteur.

\section{Références}

1. Huck C. W., Bonn G. K. Recent developments in polymer-based sorbents for solid-phase extraction. J. Chromatogr. A $2000 ; 885$ : 51-72.

2. Hennion M.-C. Solid-phase extraction : method development, sorbents, and coupling with liquid chromatography. J. Chromatogr. A $1999 ; 856: 3-54$.

3. Pichon V. Extraction sur phase solide pour l'analyse de composés organiques. Techniques de l'ingénieur. 2006 ; décembre : P1420 1-14.

4. Delaunay N., Pichon V., Hennion M.-C. Immunoaffinity solid-phase extraction for the trace-analysis of low-molecular-mass analytes in complex sample matrices. J. Chromatogr. B $2000 ; 745$ : 15-37.
5. Alexander C., Andersson H. S., Andersson L. I., Ansell R. J., Kirsch N., Nicholls I. A., O'Mahony J., Whitcombe M. J. Molecular imprinting science and technology : a survey of the literature for the years up to and including 2003. J. Mol. Recognit. 2006 ; 19 : 106-80.

6. Sellergren B. Direct Drug Determination by Selective Sample Enrichment on an Imprinted Polymer. Anal. Chem. 1994 ; 66 : 1578-82.

7. Zi-Hui M., Qin L. Determination of degradation products of nerve agents in human serum by solid phase extraction using molecularly imprinted polymer. Anal. Chim. Acta $2001 ; 435: 121-27$

8. Chapuis F., Mullot J.-U., Pichon V., Tuffal G., Hennion M.C. Molecularly imprinted polymers for the clean-up of a basic drug from environmental and biological samples. J. Chromatogr. A 2006 ; 1135 : 127-34.

9. Mullett W. M., Dirie M. F., Lai E. P. C., Guo H., He X. A 2-aminopyridine molecularly imprinted polymer surrogate micro-column for selective solid phase extraction and determination of 4-aminopyridine. Anal. Chim. Acta $2000 ; 414: 123-31$.

10. Sanbe H., Hoshina, K., Haginaka, J. Direct injection analysis of Bisphenol $\mathrm{A}$ in serum by combination of isotope imprinting with liquid chromatography-mass spectrometry. Analyst $2005 ; 130: 38-40$.

11. Dirion B., Cobb Z., Schillinger E., Andersson L. I., Sellergren B. Water-Compatible Molecularly Imprinted Polymers Obtained via High-Throughput Synthesis and Experimental Design. J. Am. Chem. Soc. 2003 ; 125 : 15101-09.

12.Abdel-Rehim M., Andersson L. I., Altun Z., Blomberg L.G. Microextraction in packed syringe online with liquid chromatography-tamdem mass spectrometry : molecularly imprinted polymer as packing material for MEPS in selective extraction of Ropivacaine from plasma. J. Liq. Chromatogr. Relat. Technol. 2006 ; 29 : 1725-36.

13. Andersson L. I., Hardenborg E., Sandberg-Stall M., Moller K., Henriksson J., Bramsby-Sjostrom I., Olsson L.-I., Abdel-Rehim M. Development of a molecularly imprinted polymer based solid-phase extraction of local anaesthetics from human plasma. Anal. Chim. Acta 2004 ; 526 : 147-54.

14. Theodoridis G., Zacharis C. K., Tzanavaras P. D., Themelis D. G., Economou A. Automated sample preparation based on the sequential injection principle : Solidphase extraction on a molecularly imprinted polymer coupled on-line to high-performance liquid chromatography. J. Chromatogr. A 2004 ; 1030 : 69-76.

15. Lai E. P. C., Wu S. G. Molecularly imprinted solid phase extraction for rapid screening of cephalexin in human plasma and serum. Anal. Chim. Acta $2003 ; 481$ : 165-74.

16. Wu S. G., Lai E. P. C., Mayer P. M. Molecularly imprinted solid phase extraction-pulsed elution-mass spectrometry for determination of cephalexin and [alpha]-aminocephalosporin antibiotics in human serum. J. Pharm. Biomed. Anal. 2004 ; 36 : 483-90.

17.Shi Y., Zhang J.-H., Shi D., Jiang M., Zhu Y.-X., Mei S.R., Zhou Y.-K., Dai K., Lu B. Selective solid-phase extraction of cholesterol using molecularly imprinted polymers and its application in different biological samples. J. Pharm. Biomed. Anal. 2006 ; 42 : 549-55. 
18. Caro E., Marcé R. M., Cormack P. A. G., Sherrington D. C., Borrull F. Direct determination of ciprofloxacin by mass spectrometry after a two-step solid-phase extraction using a molecularly imprinted polymer. Journal of Separation Science $2006 ; 29: 1230-36$.

19. Caro E., Marce R. M., Cormack P. A. G., Sherrington D. C., Borrull F. Novel enrofloxacin imprinted polymer applied to the solid-phase extraction of fluorinated quinolones from urine and tissue samples. Anal. Chim. Acta $2006 ; 562: 145-51$.

20. Brambilla G., Fiori M., Rizzo B., Crescenzi V., Masci G. Use of molecularly imprinted polymers in the solid-phase extraction of clenbuterol from animal feeds and biological matrices. J. Chromatogr. B 2001 ; 759 : 27-32.

21. Berggren C., Bayoudh S., Sherrington D., Ensing K. Use of molecularly imprinted solid-phase extraction for the selective clean-up of clenbuterol from calf urine. J. Chromatogr. A $2000 ; 889$ : 105-10.

22.C. Crescenzi S. B., P. A. G. Cormack, T. Klein, and K. Ensing. Determination of Clenbuterol in Bovine Liver by Combining Matrix Solid-Phase Dispersion and Molecularly Imprinted Solid-Phase Extraction Followed by Liquid Chromatography/Electrospray Ion Trap Multiple-Stage Mass Spectrometry. Analytical Chemistry $2001 ; 73: 2171-77$.

23. Van Hout M.W.J., Niederländer H.A.G., De Zeeuw R.A., De Jong G.J. Ion suppression in the determination of clenbuterol in urine by solid-phase extraction atmospheric pressure chemical ionisation ion-trap mass spectrometry. Rapid Communications in Mass Spectrometry $2003 ; 17: 245-50$.

24. Yang J., Hu Y., Cai J-B., Zhu X-L., Su Q-D. A new molecularly imprinted polymer for selective extraction of cotinine from urine samples by solid-phase extraction. Anal. Bioanal. Chem. 2006 ; 384 : 761-68.

25. Venn R. F., Goody R. J. Synthesis and Properties of Molecular Imprints of Darifenacin : The Potential of Molecular Imprinting for Bioanalysis. Chromatographia $1999 ; 50: 407-14$.

26. Ariffin M. M., Miller E. I., Cormack P. A. G., Anderson R. A. Molecularly Imprinted Solid-Phase Extraction of Diazepam and Its Metabolites from Hair Samples. Anal. Chem. $2007 ; 79: 256-62$.

27. Moller K., Nilsson U., Crescenzi C. Investigation of matrix effects of urine on a molecularly imprinted solidphase extraction. J. Chromatogr. B 2004 ; 811 : 171-76.

28. Möller K., Crescenzi C., Nilsson U. Determination of a flame retardant hydrolysis product in human urine by SPE and LC-MS. Comparison of molecularly imprinted solid-phase extraction with a mixed-mode anion exchanger. Anal. Bioanal. Chem. 2004 ; 378 : 197-204.

29. Suedee R., Seechamnanturakit V., Canyuk B., Ovatlarnporn C., Martin G. P. Temperature sensitive dopamine-imprinted (N,N-methylene-bis-acrylamide cross-linked) polymer and its potential application to the selective extraction of adrenergic drugs from urine. J. Chromatogr. A 2006 ; 1114 : 239-49.

30. Haginaka J., Sanbe H. Uniform-Sized Molecularly Imprinted Polymers for 2-Arylpropionic Acid Derivatives Selectively Modified with Hydrophilic External Layer and Their Applications to Direct Serum Injection Analysis. Anal. Chem. 2000 ; 72 : 5206-10.
31.Feng S. Y., Lai E. P. C., Dabek-Zlotorzynska E., Sadeghi S. Molecularly imprinted solid-phase extraction for the screening of antihyperglycemic biguanides. J. Chromatogr. A 2004 ; 1027 : 155-60.

32. Da Costa Silva R. G., Augusto F. Sol-gel molecular imprinted ormosil for solid-phase extraction of methylxanthines. J. Chromatogr. A $2006 ; 1114: 216-23$.

33. Yin J., Wang S., Yang G., Yang G., Chen Y. Molecularly imprinted solid-phase extraction for rapid screening of mycophenolic acid in human plasma. J. Chromatogr. B $2006 ; 844: 142-47$.

34. Caro E., Marce R. M., Cormack P. A. G., Sherrington D. C., Borrull F. A new molecularly imprinted polymer for the selective extraction of naproxen from urine samples by solid-phase extraction. J. Chromatogr. B $2004 ; 813$ : 137-43.

35.Xia Y., McGuffey J. E., Bhattacharyya S., Sellergren B., Yilmaz E., Wang L., Bernert J. T. Analysis of the Tobacco-Specific Nitrosamine 4-(Methylnitrosamino)-1(3-pyridyl)-1-butanol in Urine by Extraction on a Molecularly Imprinted Polymer Column and Liquid Chromatography/Atmospheric Pressure Ionization Tandem Mass Spectrometry. Anal. Chem. 2005 ; 77 : 7639-45.

36. Caro E., Marce R. M., Cormack P. A. G., Sherrington D. C., Borrull F. Synthesis and application of an oxytetracycline imprinted polymer for the solid-phase extraction of tetracycline antibiotics. Anal. Chim. Acta 2005 ; 552 : 81-86.

37. Hu S. G., Wang S. W., He X. W. An amobarbital molecularly imprinted microsphere for selective solid-phase extraction of phenobarbital from human urine and medicines and their determination by high-performance liquid chromatography. Analyst $2003 ; 128: 1485-89$.

38. Bereczki A., Tolokan A., Horvai G., Horvath V., Lanza F., Hall A. J., Sellergren B. Determination of phenytoin in plasma by molecularly imprinted solid-phase extraction. J. Chromatogr. A $2001 ; 930: 31-38$.

39. Sanbe H., Haginaka J. Restricted access media-molecularly imprinted polymer for propranolol and its application to direct injection analysis of -blockers in biological fluids. Analyst $2003 ; 128$ : 593-97.

40. Martin P. D., Jones G. R., Stringer F., Wilson I. D. Comparison of extraction of a [beta]-blocker from plasma onto a molecularly imprinted polymer with liquidliquid extraction and solid phase extraction methods. J. Pharm. Biomed. Anal. 2004 ; 35 : 1231 - 39.

41. Xie J., Chen L., Li C., Xu X. Selective extraction of functional components derived from herb in plasma by using a molecularly imprinted polymer based on 2,2-bis(hydroxymethyl)butanol trimethacrylate. J. Chromatogr. B $2003 ; 788: 233-42$.

42. Andersson L. I., Paprica A., Arvidsson T. A highly selective solid phase extraction sorbent for pre-concentration of sameridine made by molecular imprinting. Chromatographia $1997 ; 46: 57-62$.

43. Theodoridis G., Kantifes A., Manesiotis P., Raikos N. Tsoukali-Papadopoulou $\mathrm{H}$. Preparation of a molecularly imprinted polymer for the solid-phase extraction of scopolamine with hyoscyamine as a dummy template molecule. J. Chromatogr. A 2003 ; $987:$ 103-09. 
44.Lin L.-q., Zhang J., Fu Q., He L.-c., Li Y.-c. Concentration and extraction of sinomenine from herb and plasma using a molecularly imprinted polymer as the stationary phase. Anal. Chim. Acta $2006 ; 561$ : 178-82.

45.Rashid B. A., Briggs R. J., Hay J. N., Stevenson D. Preliminary evaluation of a molecular imprinted polymer for solid-phase extraction of tamoxifen. Anal. Commun. $1997 ; 34: 303-05$.

46. Mullett W. M., Lai E. P. C. Determination of Theophylline in Serum by Molecularly Imprinted SolidPhase Extraction with Pulsed Elution. Anal. Chem. $1998 ; 70: 3636-41$.

47. Mullett W. M., Lai E. P. C. Rapid determination of theophylline in serum by selective extraction using a heated molecularly imprinted polymer micro-column with differential pulsed elution. J. Pharm. Biomed. Anal. 1999 ; $21: 835-43$.

48. Muldoon M. T., Stanker L. H. Molecularly Imprinted Solid Phase Extraction of Atrazine from Beef Liver Extracts. Anal. Chem. 1997 ; 69 : 803-08.

49. Bjarnason B., Chimuka L., Ramstrom O. On-Line SolidPhase Extraction of Triazine Herbicides Using a Molecularly Imprinted Polymer for Selective Sample Enrichment. Anal. Chem. $1999 ; 71$ : 2152-56.

50.Hu S.-G., Li L., He X.-W. Comparison of trimethoprim molecularly imprinted polymers in bulk and in sphere as the sorbent for solid-phase extraction and extraction of trimethoprim from human urine and pharmaceutical tablet and their determination by high-performance liquid chromatography. Anal. Chim. Acta 2005 ; 537 : 215-22.

51.Mullett W. M., Walles M., Levsen K., Borlak J., Pawliszyn J. Multidimensional on-line sample preparation of verapamil and its metabolites by a molecularly imprinted polymer coupled to liquid chromatographymass spectrometry. J. Chromatogr. B 2004 ; 801 : 297306.

52. Rao T. P., Kala R., Daniel S. Metal ion-imprinted polymers - Novel materials for selective recognition of inorganics. Anal. Chim. Acta 2006 ; 578 : 105-16.

53. Pichon V. Selective sample treatment using molecularly imprinted polymers. J. Chromatogr. A 2007 ; 1152 : 4153.

54. He C., Long Y., Pan J., Li K., Liu F. Application of molecularly imprinted polymers to solid-phase extraction of analytes from real samples. Journal of Biochemical and Biophysical Methods 2007 ; 70 : 133-50.

55. Sellergren B. Molecularly Imprinted Polymers : Manmade mimics antibodies and their applications in analytical chemistry. B. Sellergren (Ed.) : Elsevier, 2001.

56. Gallego-Gallegos M., Muñoz-Olivas R., Cámara C., Mancheño M. J., Sierra M. A. Synthesis of a pH dependent covalent imprinted polymer able to recognize organotin species. Analyst $2005 ; 131: 98-105$.

57. Caro E., Masque N., Marce R. M., Borrull F., Cormack P. A. G., Sherrington D. C. Non-covalent and semi-covalent molecularly imprinted polymers for selective on-line solid-phase extraction of 4-nitrophenol from water samples. J. Chromatogr. A 2002 ; 963 : 169-78.

58. Yoshizako K., Hosoya K., Iwakoshi Y., Kimata K., Tanaka N. Porogen Imprinting Effects. Anal. Chem. $1998 ; 70: 386-89$.
59. Baggiani C., Giovannoli C., Anfossi L., Tozzi C. Molecularly imprinted solid-phase extraction sorbent for the clean-up of chlorinated phenoxyacids from aqueous samples. J. Chromatogr. A 2001 ; 938 : 35-44.

60. Caro E., Marce R. M., Cormack P. A. G., Sherrington D. C., Borrull F. Molecularly imprinted solid-phase extraction of naphthalene sulfonates from water. J. Chromatogr. A $2004: 1047: 175-80$.

61.Lanza F., Hall A. J., Sellergren B., Bereczki A., Horvai G., Bayoudh S., Cormack P. A. G., Sherrington D. C. Development of a semiautomated procedure for the synthesis and evaluation of molecularly imprinted polymers applied to the search for functional monomers for phenytoin and nifedipine. Analytica Chimica Acta $2001 ; 435$ : 91-106.

62. Chianella I., Piletsky S. A., Tothill I. E., Chen B., Turner A. P. F. MIP-based solid phase extraction cartridges combined with MIP-based sensors for the detection of microcystin-LR. Biosensors and Bioelectronics $2003 ; 18$ : 119-27.

63. Piletsky S., Piletska E., Karim K., Foster G., Legge C., Turner A. Custom synthesis of molecular imprinted polymers for biotechnological application : Preparation of a polymer selective for tylosin. Anal. Chim. Acta 2004 ; $504: 123-30$.

64. Chianella I., Karim K., Piletska E. V., Preston C., Piletsky S. A. Computational design and synthesis of molecularly imprinted polymers with high binding capacity for pharmaceutical applications-model case : Adsorbent for abacavir. Anal. Chim. Acta 2006 ; 559 : 73-78.

65. Wu L., Zhu K., Zhao M., Li Y. Theoretical and experimental study of nicotinamide molecularly imprinted polymers with different porogens. Anal. Chim. Acta $2005 ; 549: 39-44$.

66. Farrington K., Magner E., Regan F. Predicting the performance of molecularly imprinted polymers : Selective extraction of caffeine by molecularly imprinted solid phase extraction. Anal. Chim. Acta 2006 ; 566 : 60-68.

67.Zhu X., Su Q., Cai J., Yang J., Gao Y. Molecularly imprinted polymer membranes for substance-selective solid-phase extraction from aqueous solutions. Journal of Applied Polymer Science 2006 ; 101 : 4468-73.

68.Zhu X., Cao Q., Hou N., Wang G., Ding Z. The preparation and the recognition property of molecularly imprinted polymer of podophyllotoxin. Anal. Chim. Acta $2006 ; 561: 171-77$.

69. Perez-Moral N., Mayes A. G. Comparative study of imprinted polymer particles prepared by different polymerisation methods. Anal. Chim. Acta 2004 ; 504 : 1521.

70. Weng C.-H., Yeh W. M., Ho K.-H., Lee G.-B. A microfluidic system utilizing molecularly imprinted polymer films for amperometric detection of morphine. Sensors and Actuators B 2007: $121: 576$.

71. Caro E., Marcé R. M., Cormack P. A. G., Sherrington D. C., Borrull F. Selective enrichment of anti-inflammatory drugs from river water samples by solid-phase extraction with a molecularly imprinted polymer. Journal of Separation Science 2005 ; 28 : 2080-85. 
72.Zurutuza A., Bayoudh S., Cormack P. A. G., Dambies L., Deere J., Bischoff R., Sherrington D. C. Molecularly imprinted solid-phase extraction of cocaine metabolites from aqueous samples. Anal. Chim. Acta 2005 ; 542 : 1419.

73. Yang G., Liu H., Wang M., Liu S., Chen Y. Chromatographic characterization and solid-phase extraction on diniconazole-imprinted polymers stationary phase. Reactive and Functional Polymers 2006 ; 66 : 57983.

74. Chapuis F., Pichon V., Lanza F., Sellergren B., Hennion M.-C. Retention mechanism of analytes in the solidphase extraction process using molecularly imprinted polymers : Application to the extraction of triazines from complex matrices. J. Chromatogr. B 2004 ; 804 : 93-101.

75.Ensing K., Berggren C., Majors R. E. LC-GC Europe 2002 ; january : 16-25.

76. Matsui J., Miyoshi Y., Doblhoff-Dier O., Takeuchi T. A molecularly imprinted synthetic polymer receptor selective for atrazine. Anal. Chem. 1995; 67 : 4404-08.

77. Chapuis F., Pichon V., Lanza F., Sellergren B., Hennion M.-C. Optimization of the class-selective extraction of triazines from aqueous samples using a molecularly imprinted polymer by a comprehensive approach of the retention mechanism. J. Chromatogr. A 2003 ; 999 : 23 33.

78. Nakamura M., Ono M., Nakajima T., Ito Y., Aketo T., Haginaka J. Uniformly sized molecularly imprinted polymer for atropine and its application to the determination of atropine and scopolamine in pharmaceutical preparations containing Scopolia extract. J. Pharm. Biomed. Anal. $2005 ; 37: 231-37$.

79. Yavuz H., Say R., Denizli A. Iron removal from human plasma based on molecular recognition using imprinted beads. Materials Science and Engineering : C 2005 ; 25 : 521-28.

80. Haupt K. Molecularly Imprinted Polymers : The Next Generation. Analytical Chemistry 2003 ; 75 : 376A-83A. 